\title{
Stochastic processes in reversing figure perception*
}

\author{
M. M. TAYLOR and K. D. ALDRIDGE $\dagger$ \\ Defence and Civil Institute of Environmental Medicine \\ 1133 Sheppard Avenue West, P.O. Box 2000, Downsview, Ontario, Canada M3M 3 B9
}

\begin{abstract}
The timing patterns of figural reversals were measured, using a stimulus figure usually seen in one of two well-defined forms. The figure was a three-dimensional surface formed by denting a flat piece of Plasticene with a table tennis ball. The surface thus formed could be seen as dented or as bubbly. Transitions between the two forms were clear and abrupt and could not be voluntarily controlled by even a practiced $O$. The timing distributions of reversals, for four Os who worked for five sessions of $36 \mathrm{~min}$ each, were found to be incompatible with theories based on satiation-like effects; neither did they conform with a simple random-walk model. A random-walk model based on a finite majority decision device with memory was found to describe the data better than either. Data from 20 90-min observation periods for each of two Os were analyzed in detail according to this model, and it was found that the gross behavioral changes that sometimes occurred from period to period could usually be accounted for by a change of exactly one unit in one or another of the three parameters of the majority detector. The mathematical description of the model is given in a separate appendix to the paper.
\end{abstract}

The phenomenon of the reversing figure has had a long history (e.g., Boring, 1942). The Necker cube and Schroeder staircase have found their way into almost every introductory psychology text. In spite of the familiarity of reversing figures, the mechanism underlying such figural reversals is not clear. Often the reversal is described as due to some form of fatigue in which the "strength" of the perception of one form declines over time, permitting a possible alternative form to emerge and replace the declining percept. After a while, this alternate also fatigues, and the newly recovered original percept is able to replace it. Repetitions of this alternation of satiation and recovery lead to the continuing sequence of figural reversals. We use the terms "satiation" and "fatigue" interchangeably without intending any explicit physiological connotation for either term. A comprehensive statement of this type of theory is given by Orbach et al (1963).

In an experiment studying the time pattern of changes in ambiguous figure perception in several modes, Taylor and Henning (1963a) found that the classical Necker cube, as well as all the other tested figures, give rise not to two different perceptual forms, but to many. The conclusion drawn from these results was that the articulation of the figure permitted many different possibilities and that over time various percepts would emerge unless they had been suppressed by prior suggestion. Taylor and Henning (1963b) had previously found suggestion to be effective in altering the range of forms perceived in the verbal transformation effect, and in the case of the Necker cube, the suggestion to which psychology students are exposed in their training is quite probably enough to restrict them to perception of only

*DCIEM Research Paper No. 887.

†Present address: Killam Earth Sciences, University of Alberta, Edmonton, Alta., Canada T6G 2E1. the two cube orientations. In the absence of such suggestion, one of the naive Ss used by Taylor and Henning (1963a) saw 22 different forms of the Necker cube in $10 \mathrm{~min}$, and some other Ss saw nearly as many. Ammons et al (1959) had previously noted the possibility of many forms in the Necker cube figure.

Inspection of the orders of report for the different forms suggested that alterations in the percept were unlikely to be due to a fatigue-like process. If fatigue were responsible for the elimination of a percept, then that percept should not recur until all the other forms which had thus far been perceived had had another turn. If, for instance, three different forms were in the repertoire, they should usually be seen in cyclic order, thus: ABCABCABC ... The A form would still be "fatigued" when the B form had satiated below the level at which $\mathrm{C}$ should be seen. In fact, the changes in percept were more commonly in pairs: ABABCBCBCACA ... Furthermore, as new forms entered the repertoire, the earlier forms (which presumably were originally easier to perceive) neither dropped out nor retained their original prominance. They might recur in their turn, participating usually in pairwise alternations with other forms in the repertoire. The coarseness of timing and the difficulty of identifying all the forms reported by the Ss precluded formal analysis of these data, but provided the impetus for the present study.

Considerable time was spent searching for some figure which would give rise to three, and only three, different percepts. Such a figure could permit a direct test of the ratio of alternations to cycles in the order of report. No such figure was found, since all figures that seemed to lead to three percepts led also to further forms. We did find forms that seemed to lead to only two alternatives, other changes being only rarely seen. With such 


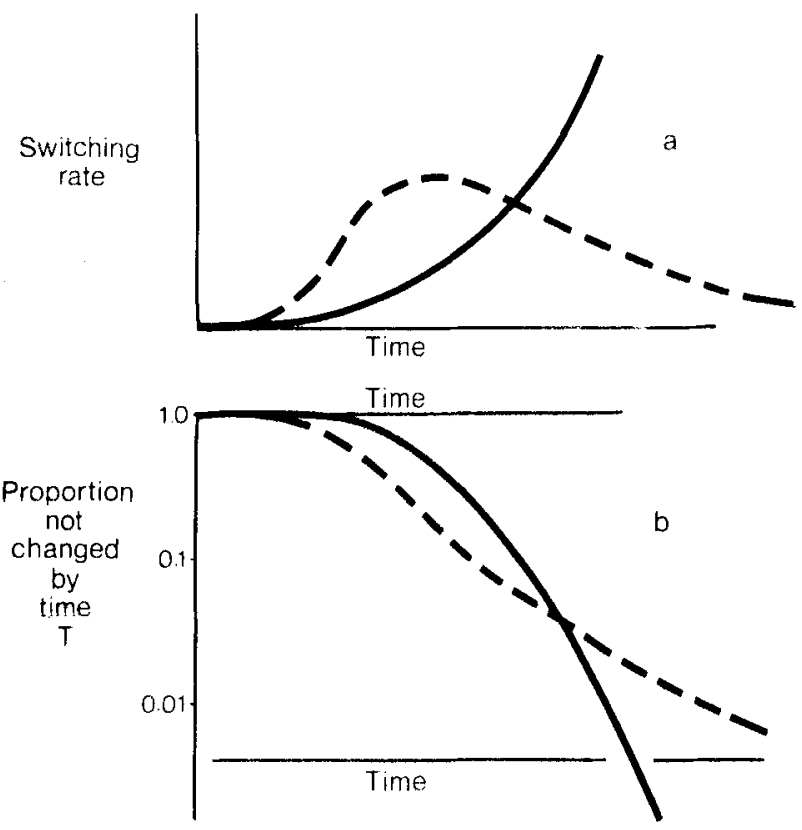

Fig. 1, Hypothetical switching rate (a) and mortality curve (b) for a fatigue model (solid curves) and for typical measurements (dashed curves) of the reversing figure effect. The upper curves (a) show the switching rate, i.e., the probability of a switch occurring in a given small time interval, as a functionof time since the last reversal of the percept. The lower curves (b) show on a. $\log$ scale the probability that no reversal has yet occurred as a function of time since the last switch. These are mortality curves, and the rate curves give their slopes, which for the fatigue models, must be always increasing as a function of time but when measured experimentally are not.

two-phase figures, we could not investigate the fatigue effect through the order in which forms were seen, but the timing of changes offered another avenue of approach.

\section{Timing of Perceptual Alternations}

The ordinary form of the fatigue hypothesis of figural alternation is that each possible percept has a "figural strength" that changes over time. The figural strength of the currently overt percept decreases, while that of any alternate possibilities increases. When the figural strength of a covert percept exceeds that of the current percept by a sufficient amount, then the covert percept becomes current, and the current one becomes covert. The figural strength of the newly current percept then begins to decline, and that of the old one, now covert, begins to increase. The statistics of the reversal timings depend on the consistency of the rates of increase and decrease in the figural strengths of the percepts.

Consider first the case in which the figural strength of the overt percept declines exponentially towards zero, while that of the suppressed percept rises exponentially toward some asymptotic value related to the accuracy with which the stimulation represents that percept. After the first reversal, subsequent reversals should occur at precisely predictable instants. The duration of a phase would depend only on the relative rates of increase and decrease of the two figural strengths and on the value of the threshold difference required between the two strengths before a reversal occurred.

Real reversals are not predictable. They are stochastically determined. If a fatigue model is to be viable, some uncertainty must be introduced into it. This can be done in at least two ways. One is to assume that the rate of fatigue is variable, as is the rate of recovery from fatigue. The second is to assume that while the fatigue and recovery rates are fixed, the reversal occurs only with a given probability if the covert percept's figural strength exceeds that of the current percept by a given amount. Of course, both forms of uncertainty may be wedded in a single fatigue model.

A useful way of visualizing the actual behavior of the switching process is to plot the probability of a change in the next $\Delta t$ (the switching rate) as a function of time since the last switch. This function, which we call the "switching rate function," but which is sometimes known as the "hazard function" among other names, is defined as $(\mathrm{dF} / \mathrm{dt})[1 /(1-F)]$, where $F$ is the probability that a reversal occurs before time $t$. According to either manner of introducing uncertainty into the fatigue model, the switching rate function rises with increasing $t$, as in Fig. 1a. Depending on the parameters of the fatigue process, the rate function may have an ever increasing slope or a slope which increases and then decreases with time; but regardless of the behavior of its slope, the overall switching rate must increase with time since the last switch.

A second function, known as a "mortality curve" is directly related to the switching rate function, and can be obtained directly from experimental data. If $F$ is the probability that a reversal occurs before time $t$, as before, then the mortality curve is given by plotting $\log (1-F)$ against $t$. The switching rate function is the negative of the slope of the mortality curve. Accordingly, for any fatigue model of the types considered here, the mortality curve must have an ever increasing downward slope, like the solid curve in Fig. 1b.

This line of reasoning has been followed by Martin (1967, 1971), who has reported data that look like the dashed curves in Figs. $1 \mathrm{a}$ and $1 \mathrm{~b}$. These results are incompatible with fatigue theories. Borsellino et al (1972), on the other hand, have found by trial and error that a two-parameter gamma distribution of ten provides a reasonable fit to the reversal periods of a Necker cube. Fox and his associates (Fox \& Herrmann, 1967; Blake, Fox, \& McIntyre, 1971) have shown similar statistics to govern the alternations of binocular rivalry. In the present experiment, we report further data which, like Martin's, seem to be incompatible with fatigue models of figural reversal.

\section{METHOD}

A figure usually seen in one of only two forms was 
Fig. 2. A photograph of a surface of the kind used in the experiment.

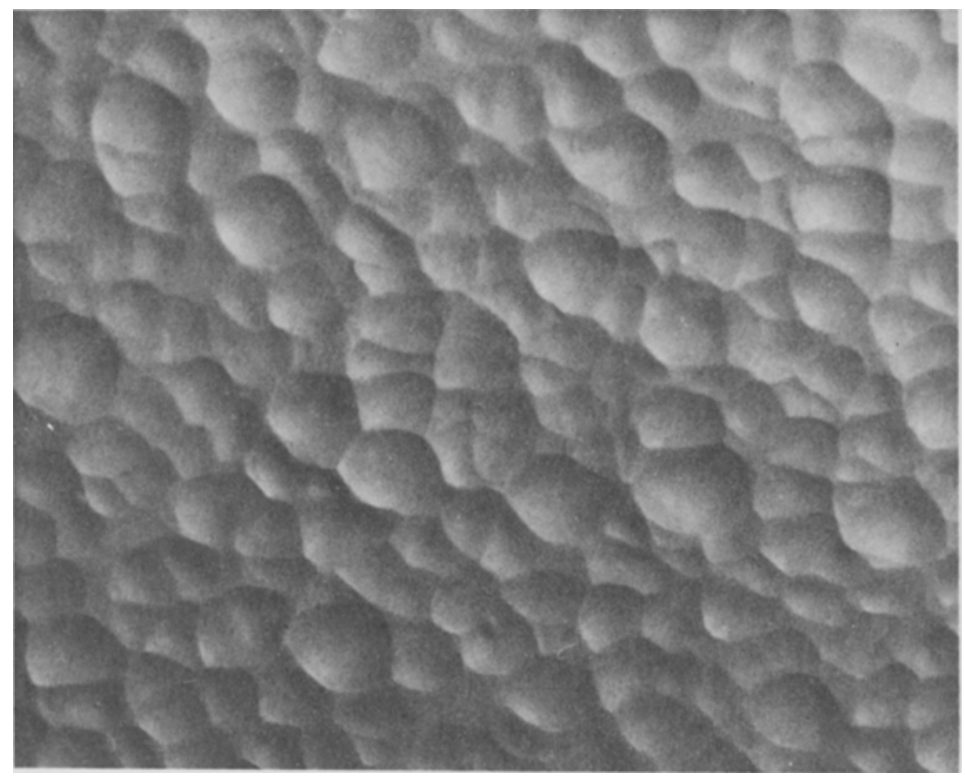

constructed by flattening a sheet of gray Plasticene and randomly denting it with a table tennis ball, making so many dents that none of the original flat surface remained. A photograph of such a surface is shown in Fig. 2. The surface appears either bubbly or dented over the whole region. Very rarely does part appear bubbly and part dented. As is common with such figures, the direction of lighting affects the initial percept, but at least with the Plasticene it does not seem to affect the reversals between dented and bubbly forms. The changes can be seen almost as easily when looking at the actual Plasticene sheet as when looking at a photograph. The stimulus figure has the further advantage that the reversals seem to be very resistant to voluntary techniques of induction. After a minute or two of observation, Os accustomed to being able to reverse other ambiguous figures almost at will found themselves unable to influence the reversals of the bubbles and dents, even by the use of various tricks, such as blinking, fixation shifts, or the interposition of other objects.

In the first experiment, the Plasticene sheet was viewed directly and binocularly by the $\mathrm{O}$ through a hole in top of a box about 18 in. deep. An 8-in.-diam circular aperture in a mask just above the Plasticene defined the field of view. A hidden horizontal $40-W$ Lumiline light illuminated the surface of the Plasticene from the side below the mask, avoiding the bias toward bubbles or dents which might be anticipated from top or bottom lighting. Even so, almost all Os saw the bubble form when first shown the display. This was unexpected, since the actual three-dimensional dented surface, not a flat photograph, was being observed. Apart from the illuminated surface of the Plasticene, the room was darkened. No fixation point was used, and the Os were encouraged to allow their viewpoint to rove over the surface. Blinking was uncontrolled and unrecorded.

The $O$ held a microswitch comfortably in one hand. She was to press the switch when she was seeing dents and to release it when she was seeing bubbles. The timings were recorded on paper tape, which was continuously being punched at 10 characters/sec. The initial percept of "bubble" was real, and not an artifact due to the switch having been open when the stimulus was first seen.

Four female Os observed for $36 \mathrm{~min}$ on each of 5 consecutive days. For two Os, the viewing time consisted of 36 1-min observation periods with 15 -sec intermissions, while for the other two, there were four 9-min observations with 3-min intermissions. The Os were housewives in their $30 \mathrm{~s}$, paid for their participation.

\section{RESULTS}

The results were analyzed in two stages. In the first stage, the simple statistics about rates of reversal and numbers of reversals per minute were calculated in the manner common for reversing figure studies. These data exhibit certain features which are interesting in their own right. In the second stage, the distributions of timing intervals between reversals were examined and compared with predictions from a model whose properties were analyzed by KDA. The analysis is described in Appendix A1 to this paper. We present first an orthodox analysis of the data, followed by a brief description of the model and some of the timing-interval data fitted by the model.

\section{First-Stage (Orthodox) Analysis}

Figure 3 shows the number of transitions per minute for each 9-min period for each of the four Os. Os $M$ and B were run for 9-min periods with 3-min breaks, while $E$ and $\mathrm{K}$ were run for 36 single-minute periods with $1 / 4-\mathrm{min}$ breaks. For $\mathrm{E}$ and $\mathrm{K}$, therefore, the split into $9-\mathrm{min}$ periods is arbitrary and is done only for convenient comparison with the data of $M$ and $B$. One may regard $E$ and $\mathrm{K}$ as having practice "distributed" on the short time scale of a few minutes, but "massed" on the scale of the day's run, whereas M and B have "massed" practice on the 9-min time scale but "distributed" practice over the day.

The four Os show characteristic differences. $K$ showed no reversals whatever until the 18 th minute of the 2 nd day, but thereafter rapidly increased the reversal rate block by block until the 4th day. In the 5 th day, she started rapidly and slowed as the day proceeded. E, on the other hand, began from the middle of the 2nd day to slow down during the day's run, while increasing her overall rate from day to day. It seems likely, though not 


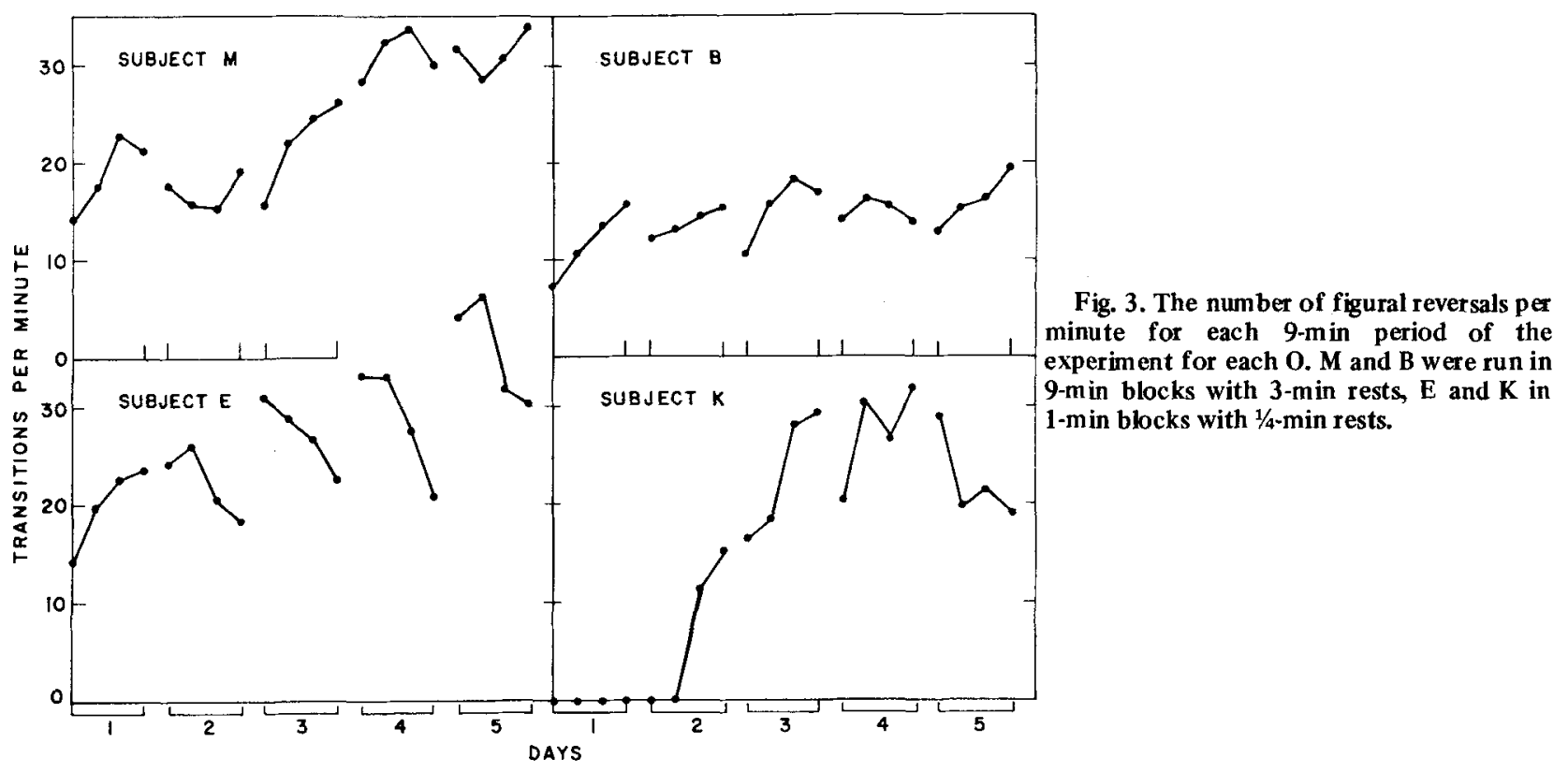

statistically defensible from these data, that Os on a semicontinuous schedule like the one given $\mathrm{E}$ and $\mathrm{K}$ would show a decline in switching rate during a run, followed by an increase after a break. This kind of effect, common in verbal learning studies when massed practice is compared with distributed, was found by Mountjoy (1961) for the size of a repeatedly tested Mueller-Lyer illusion. Ammons et al (1959) have explicitly used a Hullian learning model to describe Necker cube reversal effects, and have predicted similar differences between massed and distributed practice. Experimentally, they found a slight decline in reversal rate during the last half of a single 15 -min observation period.
Os $\mathrm{M}$ and $\mathrm{B}$, who were given continuous 9-min runs with a break between observation periods, show rises in the transition rate from block to block during each day. $M$ shows rate increases from day to day, while B shows little or no overall change in rate.

The minute-by-minute rates for the first $9 \mathrm{~min}$ of each day are shown in Fig. 4. For Os $\mathrm{M}$ and B, the data for each of the four 9-min blocks within a day were sufficiently similar to permit averaging, and these averaged minute-by-minute rates are also shown. These data show more clearly than the overall block averages the different effects of massing as against distributing practice. For $\mathrm{O} M$, in particular, massing leads to a steep decline in rate over the progress of the block, except on

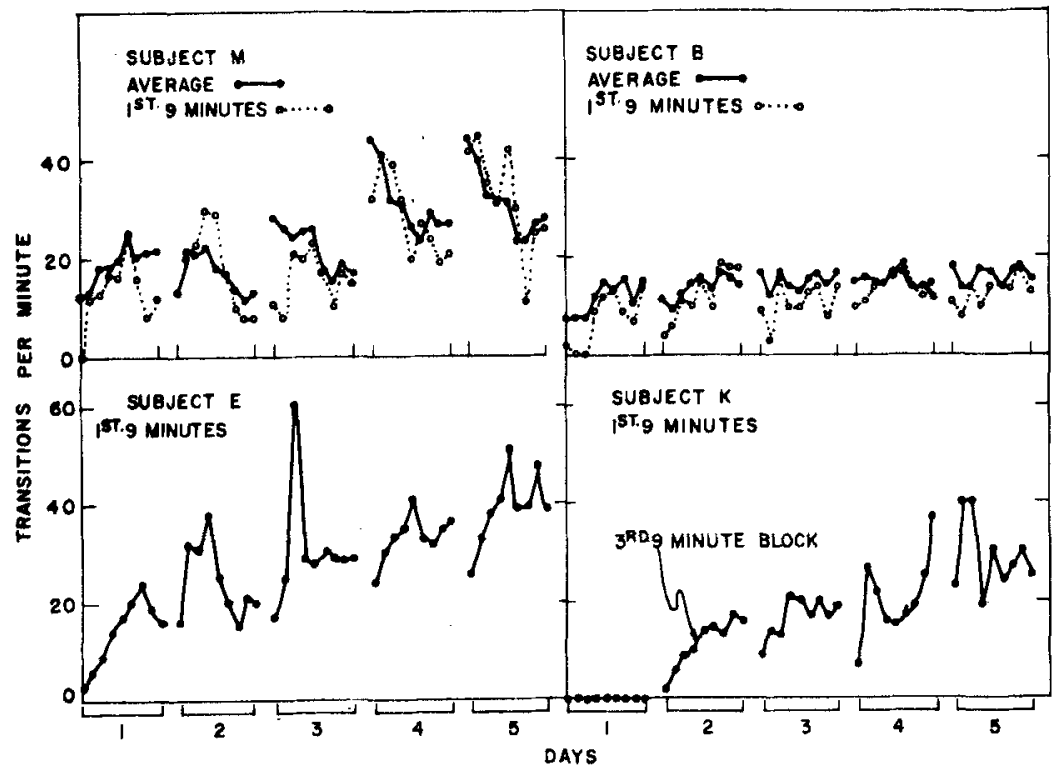

Fig. 4. Minute-by-minute fluctuation rates for the first $9 \mathrm{~min}$ of each day, and for the averaged 9-min block in the case of $M$ and $B$. 
Fig. 5. Mortality curves averaged over all data for each 0 . The probability of leaving the dent form has been plotted separately from that for the bubble form. Notice that the lessening slope of the tails could possibly be an averaging artefact, but would be contrary to the predictions of fatigue theory if real.

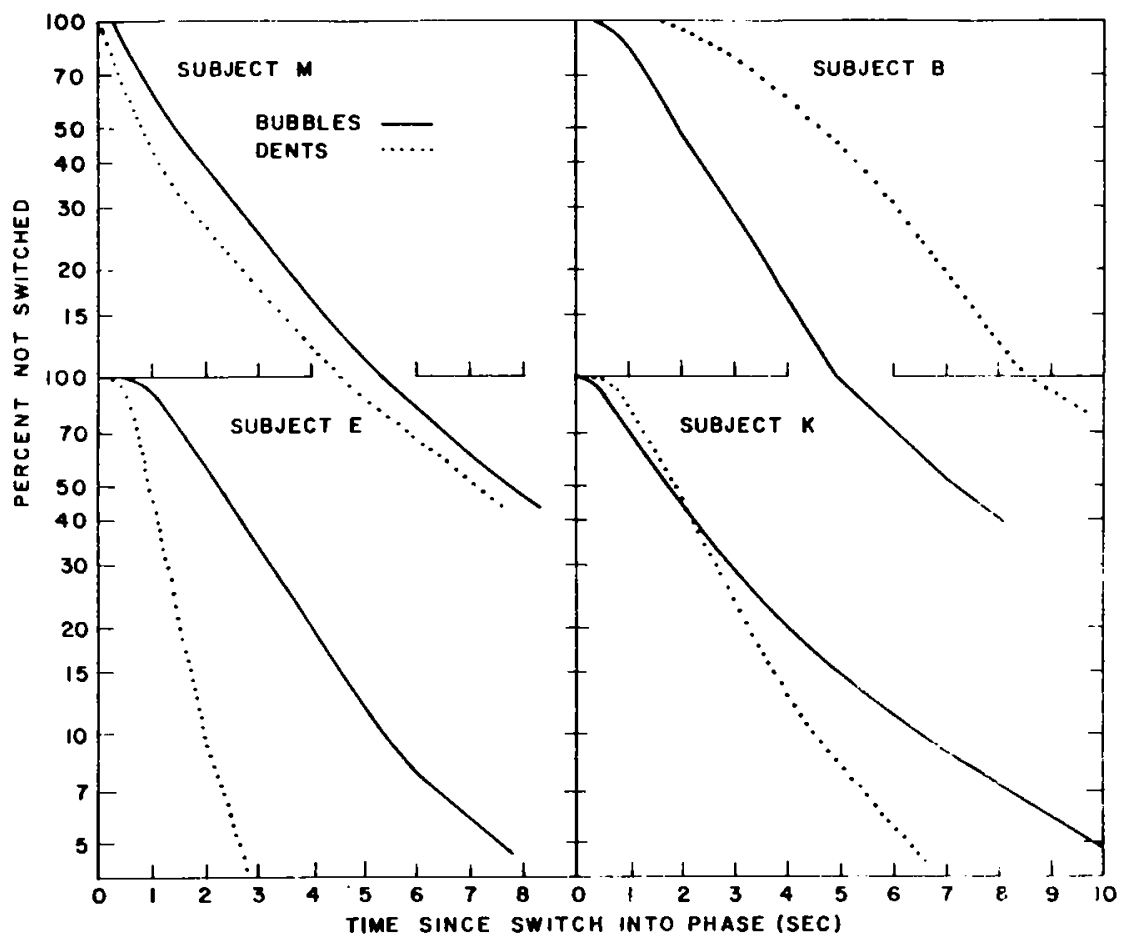

the 1st day. For $\mathrm{E}$ and $\mathrm{K}$, however, the relatively more distributed observation intervals retard the onset of the rate decline evident in Fig. 3 beyond the end of the first block. O B again shows little effect after the 1st day, and the small effect she does show is opposed to the strong effects shown by $M$. Hence, it is again improper to generalize too far from these data; the individual Os are too different.

\section{Transition Timing Results}

We now consider transitions from the bubble form to the dent form separately from the dent to bubble transitions. The Os were less idiosyncratic in these aspects of their data, which form the primary reason for this study. As primary data, we report the probability that a percept of bubble lasts longer than $T$ sec, and the corresponding probability for a percept of dent. The percentage of percepts that had not switched to the other form by $T$ sec in plotted on a logarithmic scale as a function of T. These "mortality curves," averaged over all runs, are plotted for each $\mathrm{O}$ in Fig. 5. The log scale for the mortality permits interpretation of the negative of the slope as the rate at which switching occurs, since a constant distance down the ordinate represents a constant proportion of the percepts that had not switched at the beginning of the interval being scanned. For example, it is possible, using these curves, to determine the rate at which the percept of bubble is switching for $\mathrm{OM}$ at such a time that only $25 \%$ of the original percepts are left. The rate is approximatcly $10 \%$ (of the remaining percepts) per $1 / 4 \mathrm{sec}$. We have chosen a $1 / 4 \mathrm{sec}$ as an arbitrary but convenient time unit for these rate analyses, and use it consistently in the present paper. Note that we are measuring a slope, so that the actual time unit is irrelevant except as a scale factor. The actual dimensionality of the slope measures is $\sec ^{-1}$, and a switching rate of $10 \%$ per $1 / 4 \mathrm{sec}$ can be read as $.40 \mathrm{sec}^{-1}$.

The rate of switching derived from the mortality curves is shown in Fig. 6 for each $\mathrm{O}$ as a function of the proportion of initial percepts remaining unswitched. Note that in Fig. 6, time is not the abscissa, and hence Fig. 6 is a version of the switching ratc function compressed nonlinearly horizontally. The rate is uniformly low for the first $5 \%$ of switches. This period represents the latency evident in the morality curves. According to the response data, an $\mathrm{O}$ tends to maintain his percepts for at least $1 / 4 \mathrm{sec}$. This latency may be real, or it may possibly represent a response lag indicating inability to release and press the switch faster. In either case, the rate thereafter increases to a level which remains steady or may even decrease for the longer switching intervals.

We did not test for interinterval sequential correlations. Both Martin (1971) and Borsellino et al (1972) have sought and failed to find such correlations, and it is fairly safe to assume that they are absent in the present experiment as well.

The slope provides the critical test of fatigue-like theories of figural reversal, including the Ammons ct al (1959) learning model in which reactive inhibition takes the place of fatigue. Differential fatigue theories must predict that the rate functions should increase monotonically. They should never show a region in 


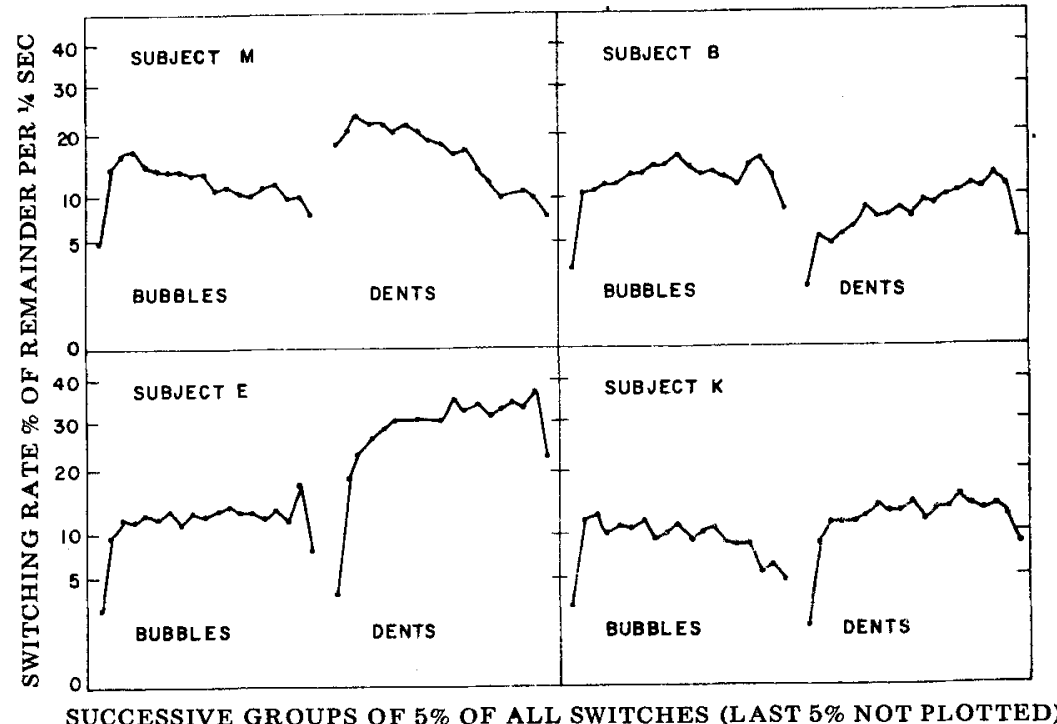

Fig. 6. Switching rates derived from the mortality curves of Fig. 5 . The rates have been determined as a function of the proportion already switched, not as a function of time. A typical point may thus be read as "at a time for which $40 \%$ of the intervals have switched, a reversal will happen with probability 0.20 in the next $1 / 4$ sec."

which the rate decreases as the time since a switch increases. Satiation or fatigue must increase with time, and increasing fatigue means increasing probability that the current percept will be replaced by its competitor. The data presented here are not compatible with any obvious model that incorporates fatigue or satiation as a major component. Martin $(1967,1971)$ came to the same conclusion. We show later that fatigue can be incorporated in a viable model, but not in a way that attributes the reversals directly to the fatigue of the form currently perceived.

The daily learning data can be reanalyzed from the viewpoint of the rate functions. The average switching rate was determined from the rate function for each separate 9-min block of data, ignoring the first and last points of the individual rate functions. These averaged rates are shown separately for bubbles and for dents in
Fig. 7, which shows a very strange phenomenon for which we have no ready explanation. Most of the changes in switching rates seem to occur in the dent phase, while the bubble phase rate does not change much from day to day. Between blocks within a day, the bubble phase for Os $\mathrm{M}$ and $\mathrm{E}$ does show small rate changes that parallel the changes in their overall transition rates (Fig. 3). But the divergence between the two phases is much more dramatic; the rate at which the dent percept changes to the bubble phase increases over days, strongly so for $\mathrm{E}, \mathrm{M}$, and $\mathrm{K}$. Note that switching rate is related to, but is not identical with the transition rates measured in the orthodox analysis.

The differences between the rates for the two phases are more clearly shown in Fig. 8 , in which the daily average rates are shown for the four Os together, the bubble phase in the first panel, the dent phase in the

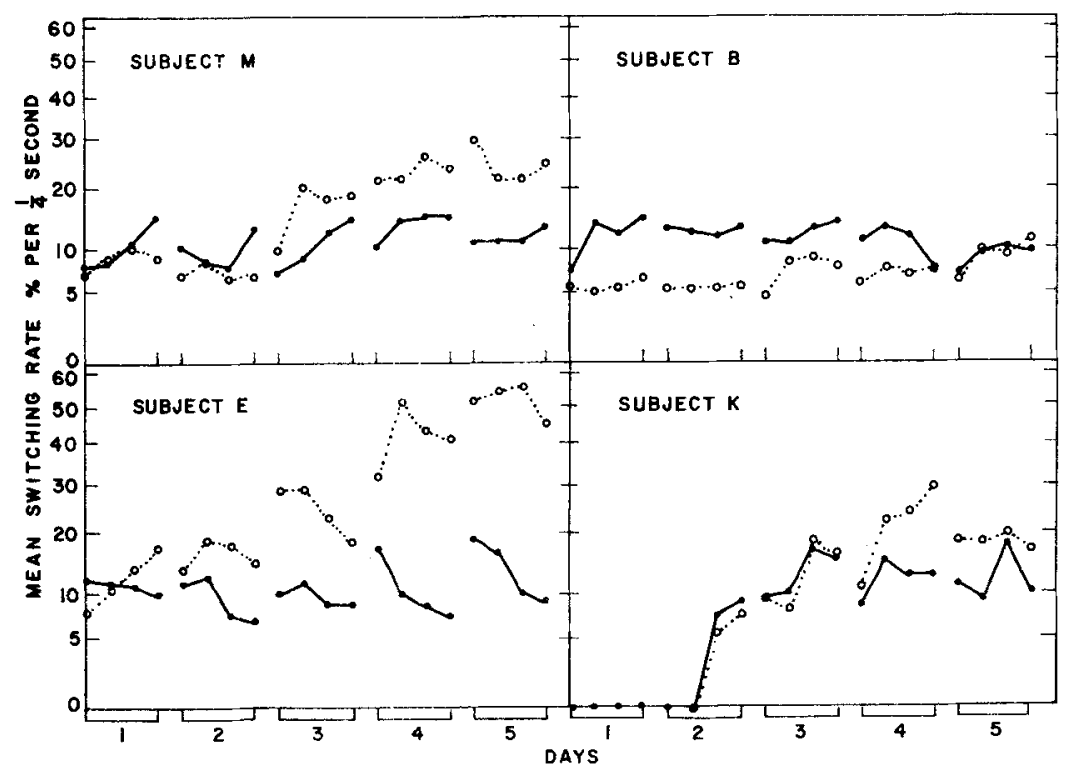

Fig. 7. Average switching rates determined from rate functions like those of Fig. 6 for each 9-min block of data. The average was determined by discarding the first and last 5\% interval of switches and averaging the rate for the middle $90 \%$. The number of switches per minute cannot be recovered from these data, since the latency interval, if any, is ignored. Bubbles are solid lines, dents are dotted. 
Fig. 8. Daily average switching rates for the four Os shown together, to indicate the lawful relationships among them and the remarkable equality over both $O$ s and days in the switching rate out of the bubble phase.

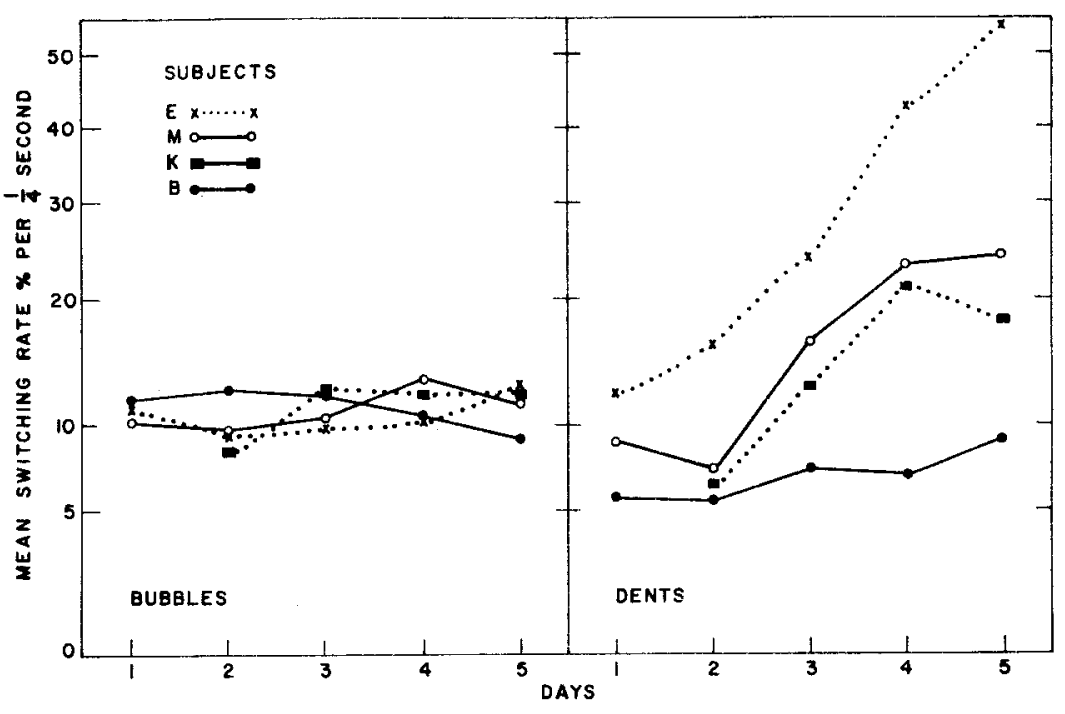

second. A remarkable feature is the equality of the bubble rates across the four Os and over days. Even O K, who showed no reversals in the first day and a half, attained the same bubble rate from her very first block of changes. The increase in dent rates is clear, as is its lawful nature. We were later unable to repeat this facet of the results using a television display instead of the direct observation of the Plasticene, and cannot offer any further suggestions. Other aspects of the data were replicable with the TV display.

\section{A STOCHASTIC MODEL}

At first sight, the data seem to be reasonably well in agreement with a purely random process, in which, after a short latency period, a switch is as likely to happen at any one moment as at any other. Martin $(1967,1971)$ used such a (pseudo-Poisson) model. If the latency period is itself somewhat variable, the characteristic mortality curve would consist of an initial flat portion curving smoothly into a linear tail. For several cases, however, the fit is rather bad. Furthermore, this model carries no indication of what significance might be attached to the consistent difference between the rate data of the two phases.

A related model that seems to have some heuristic and descriptive value was suggested by analogy with Selfridge's Pandemonium (Selfridge, 1959). Pandemonium is a device containing many "demons," each of which has the job of identifying a specific input pattern. They all shout, and the one that shouts loudest at any moment wins. The Pandemonium-decision device decides that the pattern is the one claimed by the loudest demon. In our version, each demon may choose either of two patterns, bubbles or dents. This is something of a perversion of Pandemonium, since Selfridge's demons are restricted to one decision, which they make with more or less fervour. Our demons are equally authoritative whichever decision they make, but can freely and instantaneously switch from one state to the other. The overall decision of the pattern-recognition device depends on which decision it has recently been making, so that it tends to be conservative. More demons are needed to change a decision than are needed subsequently to maintain it.

To change the metaphor, we label the demons as "cells," without presuming them to reflect individual neural cells, although we believe them to correspond to neural feature detectors at some hierarchic level. The decision device contains $r$ cells, $N_{a}$ of them in State a and $\mathrm{N}_{\mathrm{b}}=\mathrm{r}-\mathrm{N}_{\mathrm{a}}$ of them in State $\mathrm{b}$. The whole device is said to be in State $N_{a}$, defined by the number of cells in State a. Each cell switches states according to a Poisson process with Rate $\mathrm{k}$, so that the probability that it has not switched state by time $t$ is given by $\mathrm{e}^{-\mathrm{kt}}$. The device output is defined by two boundaries. If $\mathrm{N}_{\mathrm{a}}$ (the number of cells in State a, or the device's state number) is less than or equal to the lower boundary, $n$, then the device output is $\mathrm{B}$. If the state number, $\mathrm{N}_{\mathrm{a}}$, is greater than or equal to the upper boundary, $n+m$, the device output is A. If the state number lies between the two boundaries, in a "hysteresis zone" of width $m$, then the device output remains whatever it last was. As an example, the operation of such a device, with $r=20$ cells, a lower boundary $\mathrm{n}=10$, and a hysteresis zone with $\mathrm{m}=2$ is shown in Fig. 9. As time progresses, the output, which was initially in State A, switches to State B when $\mathrm{N}_{\mathrm{a}}$ declines to 10 , reverts to State A when its state number increases to 12, and so forth. The times it is in State A are denoted by $t_{A}$, and similarly $t_{B}$ denotes the times it is in State B. Only the overt output of "State A" or "State B" for the whole device is accessible to the perceiver.

Certain properties of the model are apparent from inspection. It will exhibit latency in changing its decision, because of the hysteresis zone. If the hysteresis zone is not central, then the distributions of durations of the two states will not be the same. In the example 


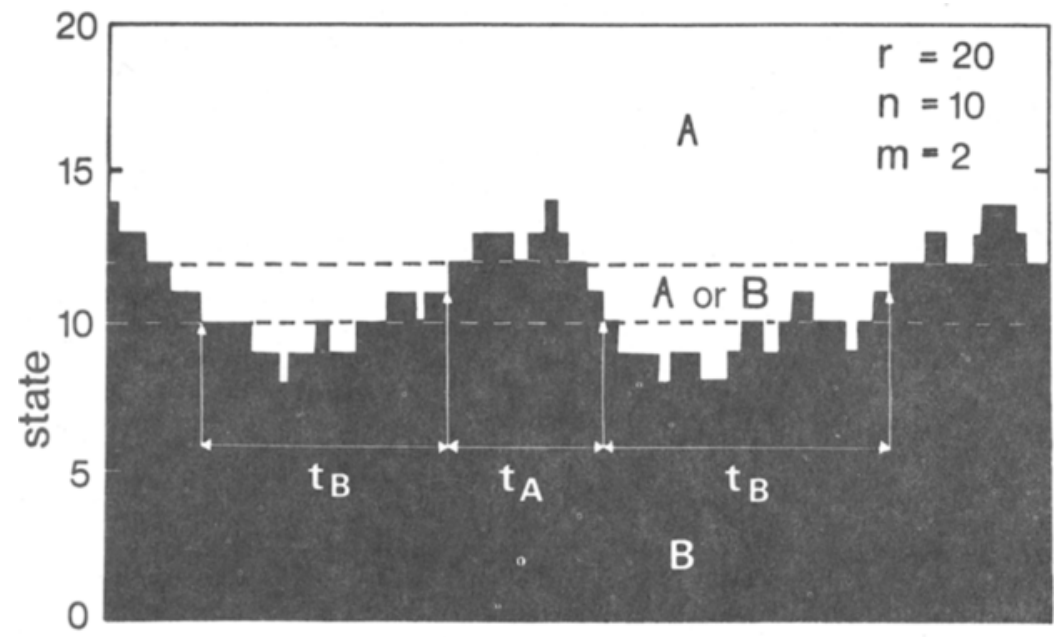

Fig. 9. A possible sequence of states in a 20-cell decision device with two possible output states, " $A$ " and " $B$. " If the number of cells reporting " $A$ " is 12 or greater, the device decides "A." If it is 10 or less, the device decides "B." And if it is exactly 11, the decision remains whatever it had been. This device is said to have a lower bound ary at 10, an upper boundary at 12 , and a hysteresis zone width of 2 . In the figure, the periods for which the device decides " $A$ " are denoted $t_{A}$, and for " $B$," $t_{B}$.

\section{time}

given, transitions out of State A will be more likely to occur quickly than will transitions out of State B, since it takes 12 cells in the a state to force an " $A$ " decision, whereas 10 cells suffice for the " $B$ " decision.

Calculating the actual distributions of times that the decision stays " $A$ " or " $B$ " is complicated. An analysis was derived by KDA and is the subject of Appendix A1 of this paper. Using this analysis, the distributions were determined for a wide range of values of $r, n$, and $m$, which are the parameters that determine the shapes of the mortality curves. The cell switching rate, $\mathrm{k}$, determines their time scale. The effects on the mortality curves and derived rate functions of varying $n$ and $m$ are shown for a 20-cell device in Fig. 10. The upper panels show the mortality curves, the lower the rate functions. Within each panel, a curve for bubbles is paired with a

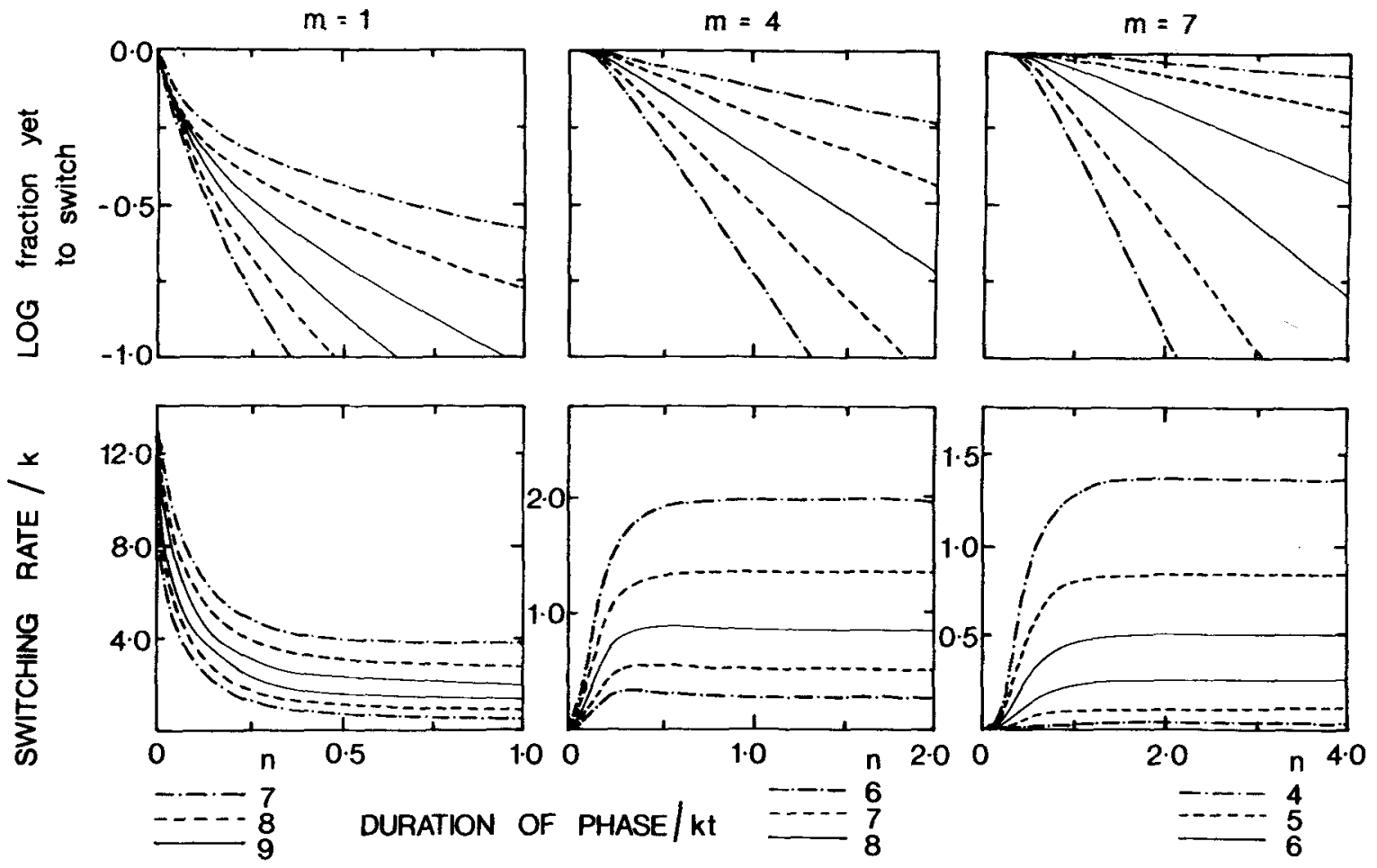

Fig. 10. Mortality curves (upper panels) and rate functions (lower panels) for decision devices with 20 cells $(r=20)$ and different positions of the hysteresis zone ( $\mathrm{n}$ is the lower bound and $\mathrm{m}$ the width of the hysteresis zone.) For each device size, one set of boundary parameters is as close to symmetrical as possible, and the others deviate from symmetry to show how the differences in the rates for the two phases may be accounted for. Time has been normalized to the unit of cell switching rate (k). The panels have different values of the hysteresis zone, 1 unit for the left pair, 4 units in the middle, and 7 units on the right. The different curves represent different degrees of symmetry, the solid lines being maximally symmetric, the dashed being 1 unit down and the dot-dashed 2 units down from that position. 
Fig. 11. Mortality curves from the model fitted to each 9-min block of data from $O B$. The parameters used in the fit are shown in the panel. The parameters are listed in order $(r, n, m)$. Each panel is $7 \frac{1}{2} \mathrm{sec}$ wide.
TRIAL

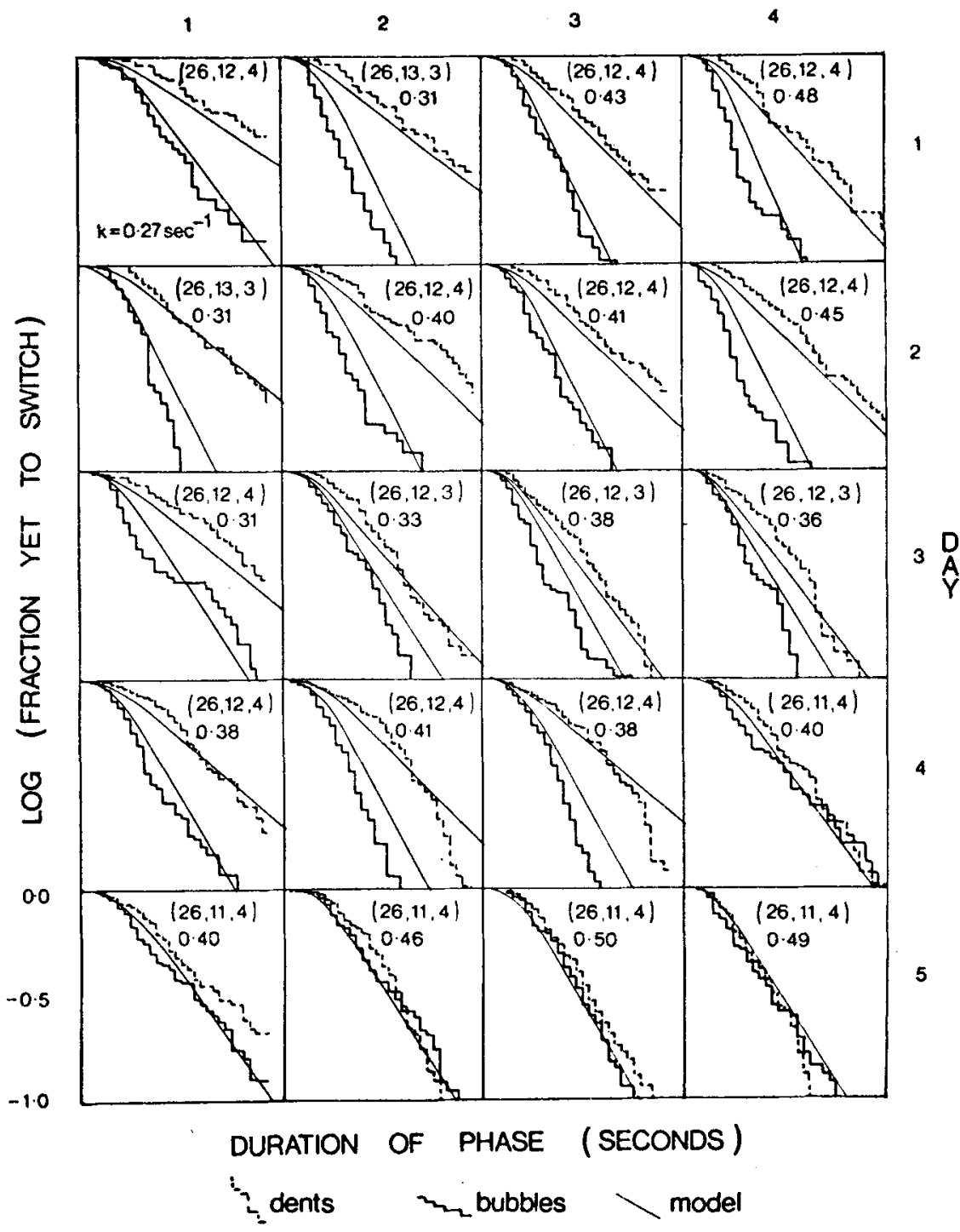

corresponding one for dents. All the time scales have been normalized in terms of $\mathrm{k}$. Across the panels from left to right, the curves are shown for increasing hysteresis zone width, and within each panel the parameter is the placement of the lower boundary, $n$, with three values chosen so that the upper boundary is (i) as close as possible to being symmetrically placed, (ii) one unit, and (iii) two units below symmetry. The divergence with departure from symmetry between the bubble state and the dent state is clear in both the mortality curves and the rate functions.

Notable features include the fact that the rate functions decline monotonically when there is no hysteresis (for $m=1$ ). Quick switches are likely, and the device shows no latency, since the first cell to switch might return the device to the original state; but if the first cell switch after a device decision change is such as to confirm the new decision, then a longer period must elapse before the decision can be reversed. Such an effect is barely discernible with hysteresis zone widths of $m=4$ or greater. In these cases, an initial latency is followed by a period of almost constant, though always decreasing, switching rate. For all values of $m$ except unity, the switching functions show an initial rise followed by a long decline.

The variations in the mortality curves with $r, m$, and $n$ are quite wide. However, there exist families of values of $\mathrm{r}, \mathrm{m}$, and $\mathrm{n}$ which give very similar mortality curves. In fitting the experimental data, the smallest satisfactory value of $r$ was chosen to prescribe which member of the family was fitted to the data. This selection was done by a technique described in Appendix A2.

Curve fitting was a long procedure, involving considerable computer time and some small exercise of personal judgment in deciding which values of the parameters to test. For this reason, the data from only 


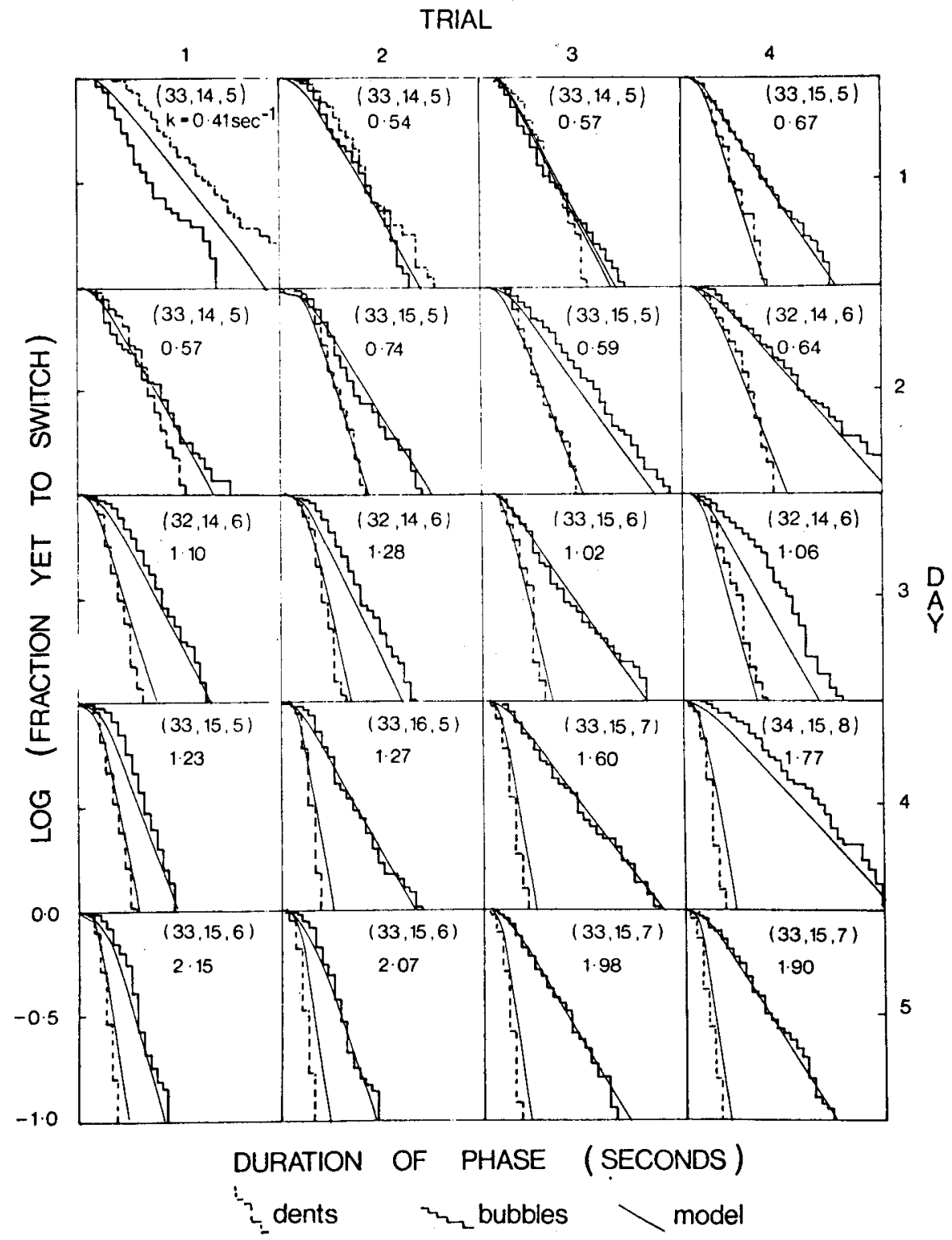

Fig. 12. Mortality curves from the model fitted to data from $\mathrm{O}$ E.

two Os were fitted. These were $B$, who showed very little variation over the 5 days of the experiment, and $E$, who showed the largest day-to-day variation and the largest divergence between the bubble phase and the dent phase (Figs. 7 and 8 ). The fitted mortality curves for each 9-min block of each day are shown for B in Fig. 11 and for E in Fig. 12. The fitting parameters are shown in each panel. Note that a single selection of parameters must fit the mortality curves for the two phases at once.

Most of the dual fits shown in Figs. 11 and 12 are reasonably close. We have no statistical estimate of the quality of the fits, because successive data points within any one graph are not independent and we know of no appropriate statistical technique. O B (Fig. 11) shows more erratic deviations than does $\mathrm{OE}$ (Fig. 12), which is somewhat surprising in view of B's day-to-day stability. For neither $O$ are there any systematic types of error, such as excess curvature, wrong tail shape, or excess latency.

The interesting feature of the parameter values used to fit the curves is their trial-to-trial and day-to-day stability in the face of considerable variation in the gross parameters of the mortality curves. These trial-to-trial shifts in the parameter values are shown graphically in Fig. 13. The abrupt changes in the mortality curves derived from the raw data are accommodated in all cases but one by a shift of no more than one unit in any parameter, such as the positions of either or both of the boundaries of the hysteresis zone or the total number of 

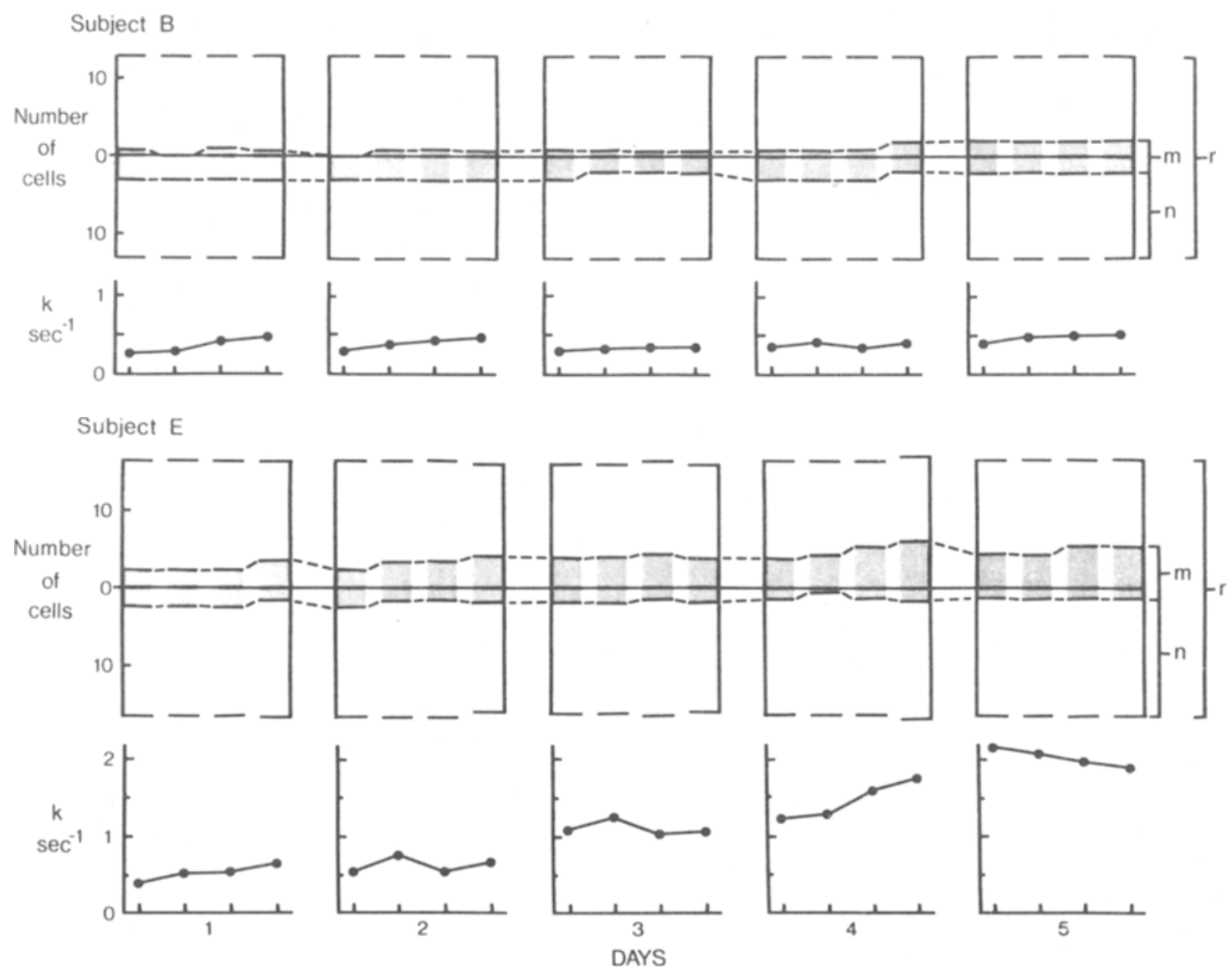

Fig. 13. Schematic depiction of the decision device parameter values used in fitting the mortality curves of Figs. 11 and 12 . The number of cells is shown by the outer boundaries, the hysteresis zone by the shaded portion. The rate parameter, $k$, is shown below the appropriate device description. $\mathrm{O} \mathrm{B}$ above, $\mathrm{O} \mathrm{E}$ below. Notice the small size of the block-to-block changes in the parameter values used to fit the appreciable behavioral changes shown by the mortality curves.

cells. The sole exception is the shift of $1 \frac{1 / 2}{2}$ units in the upper boundary for $\mathrm{O} \mathrm{E}$ between the end of Day 4 and the start of Day 5. The constancy of all the parameters except $\mathrm{k}$ (the cell switching rate, which tends to increase over time) is in marked contrast to the variability shown by any direct representation of the data, and suggests that the model may have more than mere face validity. Its ability to accommodate marked shifts in behavior is particularly well demonstrated in the abrupt change shown by $O B$ between the third and fourth run of Day 4 , where the previous discrepancy between the bubble phase and the dent phase suddenly disappears. This appreciable behavioral change is handled in the model simply by moving the whole hysteresis zone up a single unit.

This stability of the model parameters leads us to believe that the model has more than mere "fitting" validity. If some such model actually represents the mechanism of the figural reversals, then we can say that O B devotes exactly 26 feature detectors to the problem, whereas O E devotes sometimes 32 and sometimes 33 .

\section{DISCUSSION}

The data seem to contradict any theory which bases the reversal pattern of these ambiguous figures on the relative fatigue of two "figure processes." Neither do we observe the regular fluctuation of state probability found by Künnapas (1965), although it is possible that mortality curves of our type, when convolved and averaged, might predict his data. The data suggest that once a figure begins its perceptual alterations, the pattern of changes is governed by a stochastic process. On the other hand, the data are not relevant to the question of what happens during the initial latency period. Fatigue or satiation may well play a role in bringing the figure to a state in which reversals might begin. I. P. Howard (1961) has studied this initial process in detail, and his results strongly support the hypothesis that satiation is implicated. Such an initial fatigue process is entirely compatible with the stochastic model, as we now discuss. 


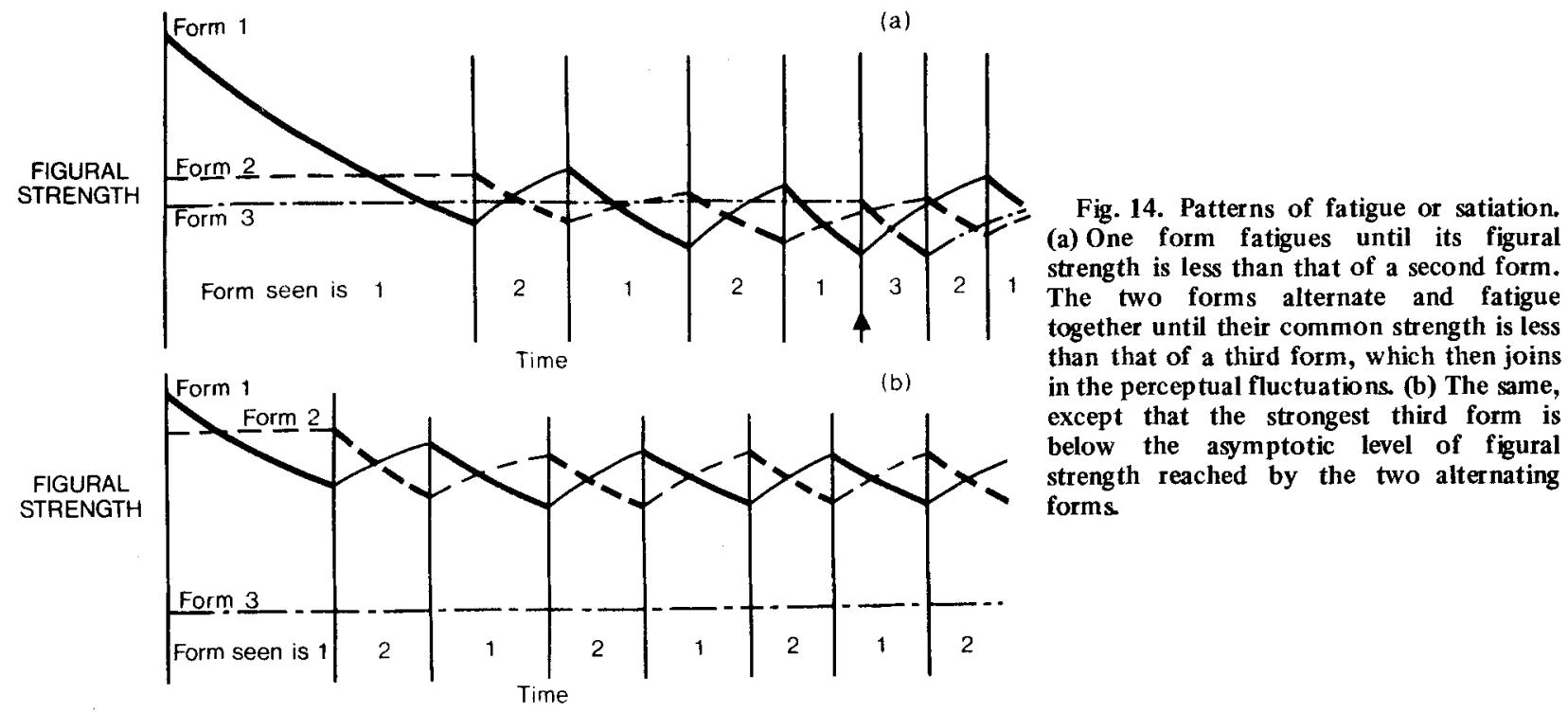

The model presented seems to be fairly satisfactory as a description of the reversal behavior of a figure with two well-defined and balanced phases. In nature, such figures must be rare, although perceptual fluctuations with prolonged observation of a simple stimulus array are so common as to be almost ubiquitous. To be useful, the model must be extended to the case of unbalanced alternatives and to figures having many perceptual possibilities.

According to the basic model used in this analysis, the individual cells have a fixed a priori probability of making either one decision or the other. The computations assume this probability to be 0.5 , which would be the case for a perfectly balanced stimulus pattern. If, however, for some stimulus pattern, it were far from 0.5 , the majority of cells would almost always be on the same side of the decision boundary, and fluctuations would hardly ever be observed. In contradiction to this prediction, almost any articulated figure does undergo frequent fluctuations of one kind or another if it is observed long enough. Sakurabayashi (1953) has found fluctuations of organization for many varieties of line patterns, and the verbal transformation effect (e.g., Warren, 1961, 1968; Taylor \& Henning, $1963 \mathrm{~b}$ ) seems to occur for any repeating verbal pattern. If the model is to accommodate these facts, then the assumption of a fixed a priori probability for each possible cell state must be dropped. Satiation may be invoked to alter the individual cell probabilities over the early phases of viewing.

Experiments in which the latency to the first reversal was timed under many conditions seem clearly to indicate the existence of something formally like the postulated fatigue effect (I. P. Howard, 1961). This can readily be brought into a more complete stochastic model of the reversal process by suggesting that each cell of the device is individually susceptible to fatigue, satiation, retroactive inhibition, or what have you. Initially, rather than being balanced between the two alternatives, an individual cell now is assumed to give a particular response with some probability greater than that of any other response. As the cell fatigues, the probability of that response declines, until it reaches that of the next most likely interpretation. In this statement, "probability" should be equated with what is often called "figural strength." At this point, the situation is as described in the simple model, with reversals for each cell determined by a random process, possibly Poisson. Satiation, according to this view, does not drive the figural reversals, but it permits them to occur.

According to the cellular fatigue model, so long as a single response is maintained by a cell, it fatigues, but when the response is superseded, it recovers. However, this recovery is presumed to be slow compared to the usual time until its next reversal, and the two cellular responses, now alternating stochastically, continue to fatigue together until their common figural strength reaches that of a third possible form, if one exists (Fig. 14a). If the recovery rate is presumed to be proportional to the depth of fatigue as compared to the initial strength of the given form, then there will be a fatigue level in which recovery of a response during an "off" period compensates for the increased fatigue during the "on" period, as shown in Fig. 14b. If such a state of asymptotic fatigue occurs, then no third form may arise, and alternations will remain restricted to two forms. This analysis suggests that all stimulus configurations should eventually lead to perceptual fluctuations between at least two forms, but not all should lead to a third form.

Generalization of the model to three or more possible forms is conceptually easy but computationally very difficult (other than by direct simulation, which is expensive). The situation for three forms is depicted in Fig. 15. If the number of cells in the three states, denoted by the position of a point within the defining triangle, is sufficiently biased to favor one form over the 
Fig. 15. The random process model applied to a situation with three possible forms. Number of cells in each state is denoted by the distance of the point from each side of the triangle. At an apex, all cells are in a single form state. (a) The device state crosses a boundary and immediately crosses back to the initial form, resulting in a quick perceptual aternation between the two forms. (b) The state crosses a boundary and then penetrates deeper into the new region, both taking time and increasing the probability that the next passage over a boundary will be into the third region. The hysteresis zone has been ignored in both parts of the figure, though it remains part of the model for multiple forms.
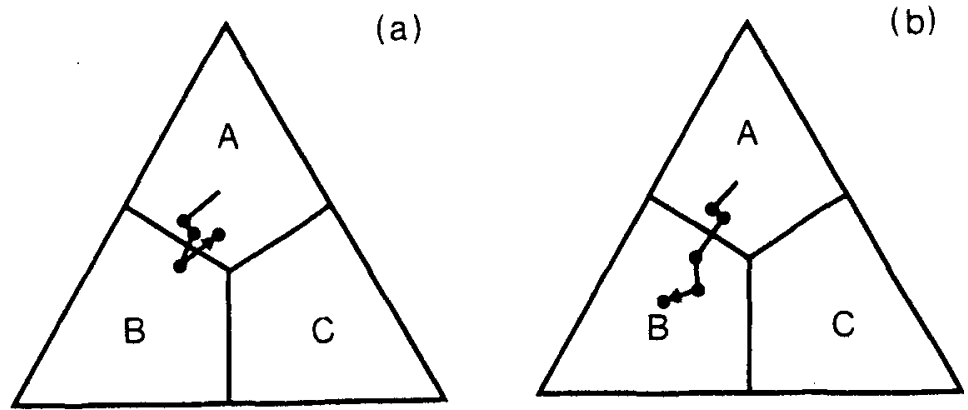

other two, the device responds with that percept. As the individual cells change state randomly among the three possibilities, the point representing the state of the device describes a random walk within the triangle. It will eventually cross a decision boundary, and the device output will change. A perceptual fluctation has occurred. With hysteresis, the boundary is now effectively moved so as to enlarge the newly entered region, enforcing a short latency period before the next fluctuation.

The multiphase model gives rise to some testable predictions of overt behavior, even though the exact computation cannot be done. The boundary most likely to be crossed shortly after a change is the one that was just crossed to make that change. It is the nearest boundary (Fig. 15a). After a longer period, the random walk has had a chance to migrate into other regions of the second state (Fig. 15b), and the probability that the next state will be different from the first one has increased. Hence, we can predict that rapid pairwise alternation will be the favored mode of fluctuation, and that a shift from pairwise alternation to a form not included in the pair should usually be preceded by a relatively long period without a change. A fatigue hypothesis would make the opposite prediction in respect of the dominance of alternations as opposed to third-form changes.

A reexamination of data from the study by Taylor and Henning (1963a) is relevant to the first part of the prediction. Considering only the Necker cube figure and its analogue, the hexagon with all diagonals drawn, and considering both binocular and monocular viewing conditions, we examined all the changes which occurred after the introduction of a third form and before the fourth was reported. For the hexagon, repetitions of the preceding form outnumbered changes to the other form by 63 to 58 , hardly a significant difference, but contradictory to the fatigue prediction nevertheless. For the Necker cube, however, repetitions dominated changes to the third form 171 to 59 . These numbers support the stochastic process model and contradict the fatigue class of theories. We do not have data which bear on the latency part of the prediction for third-form transitions as opposed to pairwise alternations.

Oyama (1961) reported that for a figure-ground reversal, the rate of reversals was lower when one of the possibilities predominated as figure. According to our model, predominance of one aspect could be due to an asymmetry in the position of the hysteresis zone. The actual transition rate is determined both by the numbers of cells in the various zones and by the cell switching rate, so that the model does not predict explicitly any relation between rate of reversal and asymmetry. If, however, the individual cell switching rate $(k$, in the model) were to be held constant, then less symmetric hysteresis zones would lead to slower overall reversal rates and greater dominance of one form. It follows that if zone asymmetry is statistically not related to cell switching rate, then there should be a negative correlation between the asymmetries (i.e., dominances) and the reversal rates reported by a population of Os. Oyama's report is in the form of just such a negative correlation.

Borsellino et al (1972) fitted gamma distributions to their reversal interval data. Fox and his associates (Fox \& Herrmann, 1967; Blake, Fox, \& McIntyre, 1971) have similarly treated the alternation periods in binocular rivalry. There is some similarity between binocular rivalry and the rivalry of ambiguous figures. In each case, one of two alternative forms may be seen, and perception of the one excludes perception of the other. Blake et al (1971) demonstrated that binocular rivalry must be a central phenomenon, as must be the reversals of ambiguous figures. The gamma fits of both Borsellino et al (1972) and Fox and associates have a common feature which further suggests something in common between the effects. All the fits displayed by both groups show an exaggerated and often early peak followed by a depressed tail when compared with the best fit gamma distribution. These deviations are not large enough to cause problems with statistical significance on any one fit, but they are consistent across the published fits. It is interesting further to note that the interval distributions implied by the model presented here deviate in just the same way from the gamma distribution.

\section{CONCLUSION}

There are many possible alternative forms of random process model that will have characteristics similar to the one analyzed here. For example, the individual cell 
switches may not conform to a Poisson process, but may be influenced by cell satiation of the kind discussed above, which would tend to equalize the periods a single cell spent in any one state. Unless this equalization were fairly exact, the averaging process performed by the decision device would make it difficult to distinguish the resulting time sequences from those generated by a purely random process. This effect is similar to that which occurs when one generates a pseudorandom noise by adding together many sinusoids. The resultant can be told from a truly random noise only by careful analysis.

A related variation in the model derives the observed asymmetry between the phases from asymmetry in the individual cell probabilities rather than from shifts in the position of the hysteresis zone. Satiation could result in such an asymmetry, if one form had a greater inherent "figural strength" than the other, since the recovery of a cell from adaptation of the stronger form would be more rapid than would its recovery from the other form.

The reintroduction of fatigue as a driving mechanism for the individual cell changes does not alter the status of the model as a stochastic model, since the randomness derives from the timing relationships among changes in the individual cells. It is this randomness that enables the model to account for the various features of the reversals observed. Only when the cells are correlated in their actions do observable fatigue-like events occur. It seems altogether likely that the individual cells are susceptible to satiation, and that a majority decision device has evolved which provides the perceived world with the stability it might otherwise lack. Such devices fall fairly into the mainstream of current pattern recognition theory.

\section{APPENDIX A1}

\section{by K. D. Aldridge}

Some properties and definitions of a stochastic model for the perception of reversing figures have been discussed earlier in this paper. It is the purpose of this section to obtain probability density functions for the random variables $t_{A}$ and $t_{B}$, the model's counterparts of percept durations.

We introduce some nomenclature for our subsequent discussion of the random process. The time taken for the process to go for the first time from State $N_{a}=n$ to State $N_{a}=n+m$ is a random variable called the first-passage time. This time is characterized by a so-called first-passage probability density function written as $f_{n, n+m}(t)$. If $N_{a}=n$ and $N_{a}=n+m$ are the lower and upper bounds of the hysteresis zone, the first-passage time characterized by $f_{n, n+m}(t)$ is just $t_{B}$, the time spent in State $B$; similarly, the time $t_{A}$ is characterized by $f_{n+m, n}(t)$, where in both cases $(0 \leqslant n$ $\leqslant r-1)$ and $(1 \leqslant m \leqslant r-n)$. The dependence of the density functions on the number of "cells," $r$, and the basic switching rate, $\mathrm{k}$, is made implicit in the definition of the functions in order to simplify notation. Each pair of first-passage probability density functions requires specification of the four parameters, $n, m, r$, and $k$. We wish to determine the family of first-passage probability density functions.

The starting point of the analysis is the observation that the next to be occupied by the random process depends on the state presently occupied. If the process is in State $N_{a}$, there are precisely $r-N_{a}$ cells which are in the " $b$ " condition and may therefore change so as to increase the state to $\mathrm{N}_{\mathrm{a}}+1$, at an overall rate $\left(\mathrm{r}-\mathrm{N}_{\mathrm{a}}\right) \mathrm{k}$. Similarly, there are $\mathrm{N}_{\mathrm{a}}$ cells which can change so as to decrease the state to $\mathrm{N}_{\mathrm{a}}-1$ at an overall rate of $\mathrm{N}_{\mathrm{a}} \mathrm{k}$ (the overall rate for some change is the sum of these two rates, rk, as it must be when $r$ independent cells switch at Rate $k$ ). Since from any State $N_{a}$, both the rate of increase to State $\mathrm{N}_{\mathrm{a}}+1$ and the rate of decrease to State $\mathrm{N}_{\mathrm{a}}-1$ are independent of time, the random process is Markovian. We shall make use of some of the mathematical techniques that have been developed for processes of this type.

For our continuous-time discrete-state process, it is expedient to introduce the Laplace transform of the first passage probability density function:

$$
f_{n, n+m}^{r}(s)=\int_{0}^{\infty} f_{n, n+m}(t) e^{-s t} d t,
$$

where the $r$ and $k$ dependence is again implicit in $f_{n, n+m}^{T}(s)$. Since the switching rate for each of the $r$ bits is $k$ for either the " $a$ " condition or the " $b$ " condition, the first-passage time from State $n$ to State $n+m$ is equal to the symmetrically equivalent first-passage time from State $r-n$ to State $r-(n+m)$. By symmetry, therefore,

$$
f_{n, n+m}(t)=f_{r-n, r-(n+m)}(t),
$$

so that the definition (Eq. 1) may refer to either member of a pair of first-passage probability density functions, by writing the appropriate subscripts. In what follows, we shall refer only to one member of this pair of functions.

Our procedure is to find an expression for $f_{n, n+m}^{T}(s)$ from the properties of the model and then invert this transform to recover $f_{n, n+m}(t)$. It can be shown by means of signal flow graphs (R. A. Howard, 1971) that first-passage transforms which are consecutive in $n$ are related recursively:

$$
f_{n, n+1}^{T}(k s)\left[(s+r)-n f_{n}^{T}-1, n(k s)\right]=r-n .
$$

(It is convenient to introduce the rate $\mathrm{k}$ into the expressions in the transform domain at this point.)

Alternatively, this recursion in the transform domain may be established by the following argument in the time domain. The transition from State $n$ to State $n+1$ will occur for the first time either directly through a transition of a " $b$ " cell to the " $a$ " state or indirectly through a change of one or more "a" cells followed by compensating " $b$ " cell changes. Hence, the mean first passage time for the process to go from State $n$ to State $n+1$ is the sum of two components: (i) the mean time for a " $b$ " cell to switch, times the chance of this occurring first, and (ii) the mean of the time for an "a" 
cell to switch, making the system go from State $n$ to State $n-1$ for the first time, followed by switches leading from State $n-1$ to State $n$ and from State $n$ to State $n+1$ the first time, times the chance of the initial "a" switch occurring first. In symbols, this statement becomes

$$
E\left(t_{n, n+1}\right)=E\left(t_{p}\right) \frac{r-n}{r}+E\left(t_{p}+t_{n-1, n}+t_{n, n+1}\right) \frac{n}{r}
$$

where $t_{i, j}$ is the first passage time random variable from State $i$ to State $j$, and $t_{p}$ is the random variable of the time for any cell to switch, an exponential of Rate rk. The application of this argument to higher order moments leads to the relationship

$$
\begin{aligned}
E\left(e^{-s t_{n, n+1}}\right)= & E\left(e^{-s t_{p}}\right) \frac{r-n}{r} \\
& +E\left[e^{-s\left(t_{p}+t_{n-1, n}+t_{n, n+1}\right)}\right] \frac{n}{r}
\end{aligned}
$$

after appropriate multiplication by powers of $s$ and summation over both sides of each equation. Integration and identification of the resulting Laplace transforms leads to Eq. $2 .^{2}$

The nonlinear recursion of Eq. 2 is converted to a linear form

$$
(r-n) U_{n+1}(k s)-(s+r) U_{n}(k s)+n U_{n-1}(k s)=0
$$

by defining

$$
f_{n, n+1}^{T}(k s)=\frac{U_{n}(k s)}{U_{n+1}(k s)} \quad n=0,1, \cdots, r-1 .
$$

$\mathrm{U}_{\mathbf{n}}(\mathrm{ks})$ is a function for which we must discover an explicit form.

An explicit expression for $U_{n}(k s)$ is found by transforming Eq. 3 into a first-order differential equation in $\tilde{U}(Z)$, defining the geometric transformation

$$
\tilde{\mathrm{U}}(\mathrm{Z})=\sum_{j=0}^{\infty} U_{j} Z^{j}
$$

where the $s$ dependence has been made implicit in both $\mathrm{U}_{\mathbf{j}} \equiv \mathrm{U}_{\mathbf{j}}(\mathrm{ks})$ and $\tilde{\mathrm{U}}(\mathrm{Z}) \equiv \tilde{\mathrm{U}}(\mathrm{Z}, \mathrm{ks})$ for simplicity of notation. Although $U_{j}$ is meaningful only for $j \leqslant r$ because there are $r$ states in this model, it is useful to define $\tilde{U}(Z)$ for all positive $\mathbf{j}$ and later retain only those $U_{j}$ for $j \leqslant r$ in the resultant expression for $\tilde{U}(Z)$.

It is readily shown by multiplying the recursion (Eq. 3) by $\mathrm{Z}^{\mathbf{n}}$ and summing over all positive integers $\mathrm{n}$ that the required $\dot{U}(Z)$ is a solution to the equation

$$
\frac{d}{d Z} \tilde{U}+P \tilde{U}=Q
$$

where

$$
P=\left[Z^{2}-(s+r) Z+r+1\right] / Z\left(Z^{2}-1\right)
$$

and

$$
\mathrm{Q}=(\mathrm{r}+1) / \mathrm{Z}\left(\mathrm{Z}^{2}-1\right)
$$

Separation of the variables $\tilde{U}, Z$, and successive integration by parts allows us to write

$$
\begin{aligned}
\tilde{U}(Z)= & \frac{1}{1-Z}+\frac{Z}{(1-Z)^{2}} \cdot \frac{s}{r}+\frac{Z^{2}}{(1-Z)^{3}} \cdot \frac{s(s+2)}{r(r-1)}+\cdots \\
& +\frac{Z^{r}}{(1-Z)^{x+1}} \cdot \frac{s(s+2) \cdots[s+2(r-1)]}{r(r-1) \cdots 3 \cdot 2 \cdot 1}+I,
\end{aligned}
$$

where $I$ is an indefinite integral having $Z^{r+1}$ as its lowest power of $Z$. Since we are concerned with only those $U_{n}$ for which $n \leqslant r$, it is unnecessary to invert that part of $\tilde{U}(Z)$ corresponding to I. From the identity

$$
\frac{Z^{i}}{(1-Z)^{i+1}}=\sum_{n=i}^{\infty}\left(\begin{array}{c}
n \\
i
\end{array}\right) Z^{n}
$$

the first $r+1$ terms of the geometric transform $U(Z)$ may be inverted by inspection to give

$$
\begin{aligned}
\mathrm{U}_{n}(\mathrm{ks})= & \left(\begin{array}{l}
n \\
0
\end{array}\right)+\left(\begin{array}{l}
n \\
1
\end{array}\right) \frac{s}{r}+\left(\begin{array}{l}
n \\
2
\end{array}\right) \frac{s(s+2)}{r(r-1)}+\cdots \\
& +\left(\begin{array}{l}
n \\
n
\end{array}\right) \frac{s(s+2) \cdots[s+2(n-1)]}{r(r-1) \cdots(r-n+1)} \\
= & 1+\sum_{j=1}^{n}\left(\begin{array}{c}
n \\
j
\end{array}\right) \prod_{i=0}^{j-1} \frac{s+2 i}{r-i} \quad n \leqslant r,
\end{aligned}
$$

where we have replaced the $s$ variable explicitly in $\mathrm{U}_{n}(\mathrm{ks}) . \mathrm{U}_{\mathrm{n}}(\mathrm{ks})$ is the coefficient of $\mathrm{Z}^{n}$ in the expression for $U(Z)$. Direct substitution of the above expression for $U_{n}(k s)$ into Eq. 3 confirms that $U_{n}(k s)$ is a solution and hence provides a solution for $\mathrm{f}_{\mathbf{n}, \mathbf{n}+\mathbf{1}}^{\mathbf{T}}(\mathrm{ks})$. This verification is somewhat simplified by noting that

$$
n\left(\begin{array}{c}
n-1 \\
j-1
\end{array}\right)=(n-j+1)\left(\begin{array}{c}
n \\
j-1
\end{array}\right)=j\left(\begin{array}{l}
n \\
j
\end{array}\right) .
$$

From Eq. 4, it is now possible to write directly an expression for the Laplace transform of the first-passage

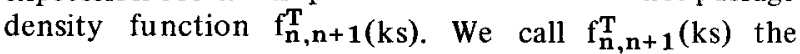
one-step transform.

To obtain an expression for $f_{n, n+m}^{T}(k s)$, the m-step transform, we reconsider the real-time random process. Since the first-passage times between consecutive states are independent random variables (the process must go from State $n$ to State $n+1$ for the first time before going from State $n+1$ to State $n+2$ for the first time), the first-passage density function for a sequence of $\mathrm{m}$ states is the m-fold convolution of the consecutive first-passage density functions. The transform of the $m$-fold convolution is the product of the $m$ consecutive transforms so that

$$
\begin{aligned}
\mathrm{f}_{\mathbf{n}, \mathrm{n}+\mathrm{m}}^{\mathbf{T}}(\mathrm{ks})= & \mathrm{f}_{\mathrm{n}, \mathrm{n}+1}^{\mathrm{T}}(\mathrm{ks}) \times \mathrm{f}_{\mathrm{n}+1, \mathrm{n}+2}^{\mathbf{T}}(\mathrm{ks}) \times \\
& \cdots \times \mathrm{f}_{\mathrm{n}+\mathrm{m}-1, \mathrm{~T}+\mathrm{m}}^{\mathbf{T}}(\mathrm{ks})
\end{aligned}
$$


From Eq. 4, this conveniently reduces to

$$
\mathrm{f}_{\mathbf{n}, \mathrm{n}+\mathrm{m}}^{\mathbf{T}}(\mathrm{ks})=\frac{\mathrm{U}_{\mathrm{n}}(\mathrm{ks})}{\mathrm{U}_{\mathrm{n}+\mathrm{m}}(\mathrm{ks})} .
$$

The m-step transform is, of course, undefined if $n+m>$ r.

We now consider the recovery of the function $f_{n, n+m}(t)$ from the transform $f_{n, n+m}^{T}(k s)$. Since the poles of this transform are located at the zeros of $U_{n+m}(k s)$, it is necessary to examine the behavior of $\mathrm{U}_{\mathrm{n}+\mathrm{m}}(\mathrm{ks})$.

Properties of $U_{n}(k s)$ for $(0 \leqslant n \leqslant r)$ are most accessible from the recursion (Eq. 3). Since from Eq. 5, $\mathrm{U}_{\mathrm{o}}(\mathrm{ks})=1$, it follows directly from $\mathrm{Eq} .3$ that $\mathrm{U}_{\mathrm{n}}(\mathrm{ks})$, written as a function of $(s+r) / r$ is an even or odd function with $\mathrm{n}$ even or odd, respectively. From Eq. 5, $U_{n}(k s)>0$ for $s>0$. Written as a function of $(s+r) / r$, $U_{n}(k s)>0$ for $(s+r) / r>1$; hence, there are no sign changes in $U_{n}(k s)$ for $(s+r) / r<-1$, because $U_{n}(k s)$ is either even or odd. Therefore, all the zeros of $U_{n}(\mathrm{ks})$ lie in the interval $(-2 \mathrm{kr}, 0)$. Furthermore, all the zeros of $\mathrm{U}_{\mathrm{n}}(\mathrm{ks})$ are real and distinct because the poly nomials

$$
\mathrm{U}_{\mathrm{o}}(\mathrm{ks}), \mathrm{U}_{1}(\mathrm{ks}) \cdots \mathrm{U}_{\mathrm{n}}(\mathrm{ks})
$$

form a Sturmian sequence in $(-2 \mathrm{kr}, 0)$ (Szegö, 1966). Proof of this latter statement follows by showing from the recursion (Eq. 3) that at a point $s_{o}$, where $U_{n}\left(k_{o}\right)=$ 0

$$
\begin{aligned}
& \text { (i) } U_{n-1}\left(k s_{o}\right) U_{n+1}\left(k s_{o}\right)<0 \text { and } \\
& \text { (ii) } U_{n}^{\prime}\left(k s_{o}\right) U_{n-1}\left(k s_{o}\right) \\
& =\frac{\sum_{m=1}^{n} \frac{m}{r-m+1} U_{m-1}^{2}\left(k s_{o}\right)}{n\left(\begin{array}{l}
r \\
n
\end{array}\right)}>0 .
\end{aligned}
$$

We now proceed to obtain the desired m-step first-passage density functions by inverting its transform, $\mathbf{f}_{\mathbf{n}, \mathbf{n}+\mathbf{m}}^{\mathbf{T}}(\mathrm{ks})$. Since all the zeros of $U_{n+m}(\mathrm{ks})$ are real and distinct, the function $\mathrm{f}_{\mathbf{n}, \mathbf{n}+\mathbf{m}}^{\mathbf{T}}(\mathrm{ks})$ will have only simple poles at those $(n+m)$ zeros of $U_{n+m}(k s)$. If $-s_{i}\left(s_{i}>0\right)$ is the ith zero of $U_{n+m}(k s)$, we may write by the method of partial fractions

$$
f_{n, n+m}^{\mathbf{T}}(k s)=\frac{U_{n}(k s)}{U_{n+m}(k s)}=\sum_{i=1}^{n+m} \frac{W_{i} \cdot s_{i}}{s+s_{i}},
$$

where

$$
W_{i}=U_{n}\left(-k s_{i}\right) / \frac{s_{i}}{r-i+1} \cdot \prod_{\substack{j=1 \\ j \neq i}}^{n+m} \frac{s_{j}-s_{i}}{r-j+1}
$$

We may now invert the transform $\mathrm{f}_{n, n+m}^{\mathbf{T}}(\mathrm{ks})$ by inspection to give the first-passage density function for $\mathbf{t}_{\mathbf{B}}$

$$
f_{n, n+m}(t)=\sum_{i=1}^{n+m} W_{i} k s_{i} e^{-k s} s_{i}
$$

Similarly, we may write the corresponding density function for $t_{A}$,

$$
f_{r-(n+m), r-n}(t)=\sum_{i=1}^{r-n} W_{i}^{*} k s_{i}^{*} e^{-k s_{i}^{*} t}
$$

with $-s_{i}^{*}$ the $i^{\text {th }}$ zero of $U_{r-n}(k s)$ and the weights $W_{i}^{*}$ defined as above, with $n$ and $n+m$ replaced by $r-(n+m)$ and $r-n$, respectively.

The density functions are related to the mortality curves and rate functions used in the main test by the relations:

$$
\begin{gathered}
P_{o}(t) \equiv P\left(\text { interval exceeds } t_{o}\right)=1-\int_{0}^{t_{o}} f(t) d(t) \\
\text { Rate at } t_{o}=-\frac{d}{d t} \log P_{o}(t)
\end{gathered}
$$

The mortality curve is simply $\log \mathbf{P}_{\mathbf{o}}(\mathrm{t})$.

\section{APPENDIX A2}

\section{FITTING PROCEDURE}

The solid heavy lines in Figs. 11 and 12 originate from the stochastic model. The selection of pairs of distributions from the three-parameter family $(r, n, m)$ was begun by calculating the first (smallest absolute magnitude) zero, $-s_{p}$, of each of the polynomials $U_{p}(k s)$ for $0<p<r$ over the range $r=5$ to $r=50$. This first zero gives the asymptotic slopes of any mortality curve. A table of ratios $s_{n+m} / s_{r}-n$ for each $r$ over all $(n, m)$ values then specified a unique index for every possible pair of distributions. The corresponding ratios of asymptotic slopes for each pair of experimental distributions were then tabulated. Since there are simply more theoretical ratios available as the number of cells, $r$, grows larger, there is no unique number, $r$, of cells for $\exists$ set of experimental ratios. However, we choose to select the lowest value of $r$ which will adequately provide the necessary theoretical ratios through the adjustment of $(n, m)$ to cover almost all the observed ratios. In some cases, a slight improvement in ratio selection can be found by further adjusting the number of cells by \pm 1 . Once the $(r, n, m)$ selection has been made, the rate, $k$, is automatically fixed by the actual asymptotic slope of the experimental distributions. For each $O$, a latency distribution was then chosen of the form $\lambda^{2}$ te $-\lambda t$. $\lambda$ was fixed for each $O$ over all the fits, and represents the time actually taken in pushing the button. $\lambda$ was always around 10 to $15 \mathrm{sec}^{-1}$, corresponding to a time constant of $70-100 \mathrm{msec}$. The delay thus introduced is meaningful in terms of the responding mechanism, but has little effect on the actual distributions as fitted, since they have "time constants" of the order of seconds. Its main effect is a slight interaction with the choise of the width of the hysteresis zone. The value of $\lambda$ used for each $O$ was determined by 
the average residual delay in the mortality curves after the completion of the slope-fitting procedure. The same value was used for all fits for a given $O$. Note that the implied delay is appreciably shorter than the commonly measured "reaction time." Reaction time presumably includes some time to interpret the stimulus input and a further time to generate the response following the decision that a response is required. The parameter $\lambda$ here represents only the response time portion of the reaction time, and seems to be of a reasonable order of magnitude.

The chosen sets $(r, n, m)$ are certainly not unique. We could always find a larger number of cells, for example, and obtain a set of distributions very close to those shown in Figs. 11 and 12 . If, however, we chose to keep $\mathrm{n}$ or $\mathrm{m}$ constant over all trials, very large changes in $\mathrm{r}$, and hence in $\mathrm{k}$, would be required between certain trials in order to satisfy the changes in asymptotic rate ratios. On the contrary, by keeping $r$ relatively constant, we have required only small changes in $n$ and $m$ from trial to trial to satisfy the experimental distributions. Thus, our algorithm does not provide a unique fit to experimental data; rather, it demands only minimal changes in the model parameters. Personal judgment exists in the procedure but is kept to a minimum.

\section{REFERENCES}

Ammons, R. B., Ulrich, P., \& Ammons, C. H. Voluntary control of perception of depth in a two-dimensional drawing. Prpceedings of the Montana Academy of Sciences, 1959, 19, 160-168.

Blake, R. R., Fox, R., \& McIntyre, C. Stochastic properties of stabilized-image binocular rivalry alternations. Journal of Experimental Psychology, 1971, 88, 327-332.

Boring, E. G. Sensation and perception in the history of experimental psychology. New York: Appleton-Century-Crofts, 1942.

Borsellino, A., de Marco, A., Allazetta, A., Rinesi, S., \& Bartolini, B. Reversal time distribution in the perception of visual ambiguous stimuli. Kybernetic, 1972, 10, 139-144.

Fox, R., \& Herrmann, J. Stochastic properties of binocular rivalry alternations. Perception \& Psychophysics, 1967, 2, 432-436.

Howard, I. P. An investigation of the satiation process in the reversible perspective of revolving skeletal shapes. Quarterly Journal of Experimental Psychology, 1961, 13, 19-33.
Howard, R. A. Dynamic probabilistic systems. New York: Wiley, 1971 .

Künnapas, $T$. Intensity of the underlying figural process. University of Stockholm Psychological Laboratories, Report No. $197,1965$.

Martin, J. N. T. The perception of reversible perspective figures. Papers in Psychology, 1967, 1, No. 2.

Martin, J. N. T. A nalysis of reversals of ambiguous perspective figures as time series of events. Unpublished $\mathrm{PhD}$ dissertation, University of London, 1971.

Mountioy, P. T. Intrasession decrement and intersession recovery to the Mueller-Lyer figure. Perceptual \& Motor Skills, 1961, 13, 51-57.

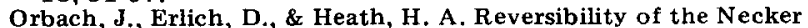
cube: I. An examination of the concept of "satiation of orientation." Perceptual \& Motor Skills, 1963, 17. 439-458.

Oyama, $T$. Relation between figure-ground dominance and reversal rate. Perceptual \& Motor Skills, 1961, 13, 438.

Sakurabayashi. H. Study in creation, IV: The meaning of prolonged inspection from the standpoint of creation. Japanese Journal of Psychology, 1953, 23, 207-216.

Selfridge, $O$. G. Pandemonium: A paradigm for learning. In The mechanisation of thought processes. London: $H . M$. Stationery Office, 1959.

Szegö, G. Orthogonal polynomials. American Mathematical Society, Vol. 23, Chap. 3, 1966.

Taylor, M. M., \& Henning, G. G. Transformations of perception with prolonged observation. Canadian Journal of Psychology, $1963 \mathrm{a}, 17,349-360$.

Taylor, M. M., \& Henning, G. B. Verbal transformations and an effect of instructional bias on perception. Canadian Journal of Psychology, 1963b, 17, 210-223.

Warren, $R$. Illusory changes of distinct speech upon repetition-The verbal transformation effect. British Journal of Psychology, 1961, 52, 249-258.

Warren, R. M. Verbal transformation effect and auditory perceptual mechanisms. Psychological Bulletin, 1968, 70, 261-270.

\section{NOTES}

1. We thank a reviewer for pointing out that fatigue models can indeed lead to gamma distributions of interreversal intervals. Our original conception of the fatigue process had incorporated only threshold uncertainty as responsible for the stochastic nature of the reversal timing, and we had therefore regarded the experimental findings of gamma-distributed reversal intervals as evidence against fatigue models.

2. We are indebted to the same reviewer for suggesting this conditioning argument to establish Eq. 2.

(Received for publication November 11, 1972; revision received February $12,1974$. 